\title{
REABILITAÇÃO CARDÍACA NA DISFUNÇÃO AUTONÔMICA: ESTUDO DE REVISÃO
}

\author{
CARDIAC REHABILITATION IN AUTONOMIC DYSFUNCTION: REVIEW STUDY
}

${ }^{1}$ Gildete da Silva Costa, ${ }^{2}$ Heloana Araújo Cruz, ${ }^{3}$ Adriana Arruda Barbosa Rezende,

${ }^{4}$ Rodrigo De Faveri Moreira, ${ }^{5}$ Elizângela Sofia Ribeiro Rodrigues.

\begin{abstract}
RESUMO
Introdução: As desordens nervosas das disfunções do sistema autônomo podem se manifestar de diversas maneiras de acordo com o sistema predominante acometido, podendo ser tanto o simpático ou parassimpático e podem acarretar alterações significativas nos dados vitais e ocasionar perdas na qualidade de vida dos pacientes. Entretanto sabe-se que a modalidade terapêutica da reabilitação cardíaca pode contribuir positivamente para minimização dos sintomas associados. Objetivo: Verificar a eficácia do tratamento de reabilitação cardíaca na disfunção autonômica. Material e métodos: Foi realizada uma revisão de literatura sistemática, comparativa e descritiva sobre os efeitos da reabilitação cardíaca na disfunção autonômica. Para inclusão na amostra foram utilizados periódicos de livre acesso, disponíveis nas bases de dados científicas Medline, Lilacs, Pubmed e Scielo, disponíveis nas línguas portuguesa e inglesa, publicados nas últimas quatro décadas. Para busca foram utilizados os descritores reabilitação cardíaca; disautonomia; sistema nervoso autônomo e seus respectivos em inglês cardiac rehabilitation; dysautonomia; autonomic nervous system. Resultados: A presença de comorbidades, mudanças corporais e idade avançada podem acarretar alterações nas respostas do sistema autonômico. Além disso, o tipo de treino e o método de avaliação empregado também podem influenciar na percepção da resposta que mantêm o equilíbrio simpatovagal. Conclusão: A atuação da reabilitação cardíaca em pacientes com disautonomia parece minimizar os sintomas deletérios associados à patologia e consequentemente pode melhorar a qualidade de vida dos portadores da doença, entretanto são necessários mais estudos para elucidar o papel da reabilitação física sobre a modulação autonômica.
\end{abstract}

Descritores: Reabilitação cardíaca; Disautonomia; Sistema nervoso autônomo.

\section{ABSTRACT}

Introduction: Nervous disorders of the autonomic system dysfunctions may manifest in different ways according to the predominant system, and may be either sympathetic or parasympathetic and may cause significant alterations in vital data and cause losses in patients' quality of life. However, it is known that the therapeutic modality of cardiac rehabilitation may contribute positively to the minimization of associated symptoms. Objective: The objective of this study was to verify in the scientific literature the existence of cardiac rehabilitation for autonomic dysfunction. Material and methods: We carried out a systematic, comparative and descriptive literature review on the effects of cardiac rehabilitation on autonomic dysfunction. For inclusion in the sample, free access journals were available, available in the scientific databases Medline, Lilacs, Pubmed and Scielo, available in the Portuguese and English languages, published in the last four decades. For the search we used the descriptors cardiac rehabilitation; dysautonomia; autonomic nervous system and their respective English-language cardiac rehabilitation; dysautonomia; autonomic nervous system. Results: The presence of comorbidities, body changes and old age can lead to alterations in the autonomic system responses. In addition, the type of training and the evaluation method used may also influence the perception of the response that maintains the sympatovagal balance. Conclusion: The performance of cardiac rehabilitation in patients with dysautonomia seems to minimize the deleterious symptoms associated with the pathology and consequently may improve the quality of life of patients with the disease, however, further studies are needed to elucidate the role of physical rehabilitation on autonomic modulation.

Descriptors: Cardiac rehabilitation; Dysautonomia; Autonomic nervous system.

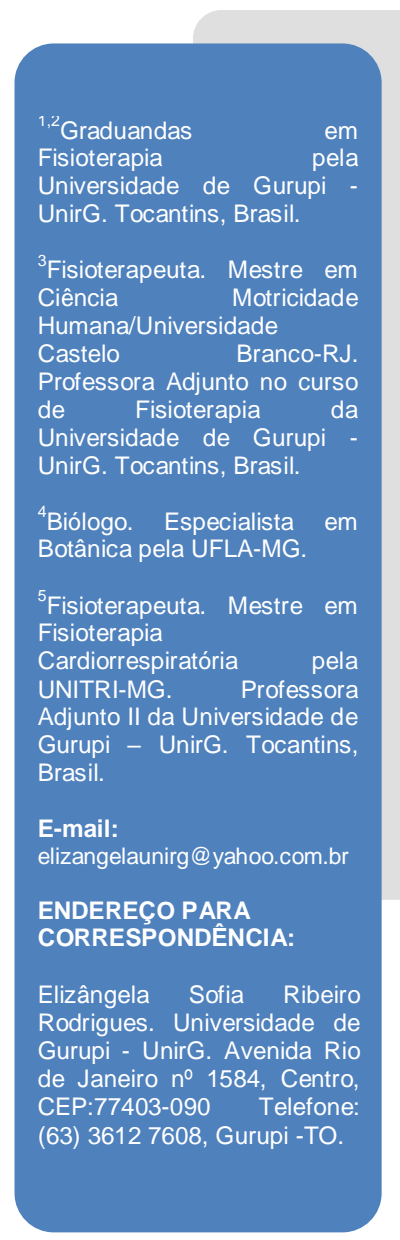

\section{INTRODUÇAOO}

O Sistema Nervoso Autônomo (SNA) controla as funções viscerais coordenadas por centros localizados no tronco cerebral e no hipotálamo, chamados de ramos simpático e parassimpático. As fibras eferentes emergem dos segmentos torácicos e lombares, ramificando no coração e vasos sanguíneos.

Em relação ao sistema cardiovascular o ramo parassimpático participa fundamentalmente no controle da frequência cardíaca através do nervo vago. 
Recebido: 07 junho 2018. Aceito: 14 dezembro 2018. Revista Amazônia Science \& Health - 2018 Out/Dez.
Costa, GS. Cruz, HA. Rezende, AAB. Moreira, RF. Rodrigues, ESR. REABILITACCÃO CARDÍACA NA DISFUNÇÃO AUTONÔMICA: ESTUDO DE REVISÃO
A disfunção autonômica surge em decorrência de um desajuste do Sistema Nervoso Simpático e do Sistema Nervoso Parassimpático. Essas duas subdivisões anátomo funcionais em perfeito estado são fundamentais para a regulação e o equilíbrio fisiológico interno do nosso organismo. ${ }^{2}$

Segundo De Angelis et al. ${ }^{3}$ conceitualmente, a fisiopatologia da disfunção autonômica despontou em meados da década de 20, a partir dos estudos de Bradbury e Eggleston que elucidaram as causas neurogênicas da hipotensão postural.

Além disso, a disfunção autonômica pode afetar diretamente a saúde de pessoas que tiveram doenças transitórias ou até mesmo doenças neurodegenerativas progressivas. As disfunções simpáticas e parassimpáticas podem ser fatores casuais ou funcionais de diferentes condições clínicas ou funcionais, podendo ser consequências secundárias de outras patologias. ${ }^{2}$

Entre as condições que podem determinar as alterações secundárias crônicas da função autonômica cardíaca incluem-se a doença de chagas, diabetes mellitus, insuficiência cardíaca, infarto agudo do miocárdio, insuficiência coronariana crônica, a hipertensão arterial e as síndromes neurológias. ${ }^{4}$

De acordo com Junqueira $\mathrm{Jr}^{2}{ }^{2}$ o diagnóstico da disfunção autonômica cardíaca está relacionado à variabilidade da frequência cardíaca (VFC), manifesta no intervalo RR do eletrocardiograma, que atuam através dos ramos simpático e parassimpático sobre o nó sinusal.

Para Rocha ${ }^{5}$ os mecanismos descritos das síndromes disautonômicas ocorrem devido ao tempo prolongado na posição ortostática, que alteram as funções do coração que se conectam ao núcleo do vago.

Em ortostatismo o retorno do sangue venoso para o coração diminui, podendo causar um decréscimo de $40 \%$ do volume de ejeção sistólico, o que pode precipitar a manifestação da disfunção autonômica. ${ }^{6}$

A posição supino resulta em respostas posturais reflexas, reguladas pelos ramos do sistema nervoso autónomo e baroreflexos arteriais e mecanoreceptores cardiopulmonares, que atuam conjuntamente para compensar a ação da gravidade sobre o volume sanguíneo, o que ocorre em cerca de 60 segundos.

A frequência cardíaca aumenta cerca de 10 a 15 batimentos por minuto, a pressão diastólica aumenta cerca de $10 \mathrm{mmHg}$, com apenas uma ligeira flutuação da pressão sistólica.

Entretanto, em condições patológicas há uma resposta inapropriada à mudança de posição corporal de decúbito para supino, ocorrendo um aumento exagerado e excessivo da frequência cardíaca na posição de pé, o que exige que o sistema cardiovascular necessite de trabalho suplementar para manter a pressão arterial e o fluxo cerebral adequados e constantes. Os sinais e sintomas da disfunção autonômica ocorrem com a postura ortostática e podem ser percebidos pela manifestação de sintomas como fraqueza, fadiga, letargia, palpitações, sudorese, distúrbios visuais (incluindo falta de sono, aumento do brilho, visão em túnel); distúrbios auditivos (incluindo deficiência auditiva, estalos e zumbidos), dor no pescoço (occipital/paracervical), dor na região das costas, dor lombar ou precordial. ${ }^{6}$

Os pacientes crônicos com desordens primárias e falência do sistema nervoso autônomo apresentam aumento da frequência cardíaca persistente ao se manterem na posição ortostática, manifestada com taquicardia de repouso e hipotensão postural, o que pode levar a síncopes e complicações associadas às quedas. As manifestações da disautonomia podem prejudicar as atividades de vida diária (AVDs) e a qualidade de vida dos indivíduos acometidos.

Willians et al. ${ }^{7}$ em estudos concluíram que a ativação relacionada com emoção, medo e raiva, aumentam o ritmo cardíaco e a pressão sanguínea, - que provoca alterações dos movimentos respiratórios.

Bassich et al. ${ }^{8}$ relataram que os sintomas da disfunção autônoma estão relacionados aos fatores hipotensão ortostática, constipação, incontinência, impotência, xerostomia, alterações visuais. Os estudiosos relataram os sinais da disfunção autônoma associados com 0 aparecimento de ataxia, incoordenação rigidez, bradicinina, tremor e labilidade emocional.

Truong et al. ${ }^{9}$ descreveram que na doença de Parkinson com sintomas típicos, os mesmos podem apresentar sintomas de disfunção autônoma, com alterações olfatórias, fadiga, dor, distúrbios do sono, depressão e demência.

Atualmente sabe-se que o controle autonômico é fundamental para um ajuste ideal nas atividades de vida diária dos pacientes. Fisiologicamente vários órgãos são controlados pelo sistema nervoso simpático e parassimpático, tais como coração, pulmões, bexiga e atividade cerebral. A ação oposta do sistema parassimpático e simpático colabora para a homeostase corporal. ${ }^{10}$

O sistema nervoso autônomo em condições normais tem a capacidade de se autorregular e se adaptar ao meio interno e externo do organismo para garantir a homeostase corporal. ${ }^{11}$

De acordo com o relatado por Lopes et al. ${ }^{12}$ estudos evidenciaram um desgaste fisiológico do corpo com o tempo, ocasionando uma diminuição da VFC relacionada a uma redução na atuação vagal sobre o nodo sinusal, que por sua vez causa um aumento da morbidade e mortalidade em idosos.

Entretanto o tratamento com atividade física pode colaborar positivamente para a redução dos sintomas associados à disautonomia. Acredita-se 
Recebido: 07 junho 2018. Aceito: 14 dezembro 2018. Revista Amazônia Science \& Health - 2018 Out/Dez.
Costa, GS. Cruz, HA. Rezende, AAB. Moreira, RF. Rodrigues, ESR. REABILITACCÃO CARDÍACA NA DISFUNÇÃO AUTONÔMICA: ESTUDO DE REVISÃO que a modalidade terapêutica específica da reabilitação cardíaca possa melhorar o desempenho cardiovascular e as respostas autonômicas, minimizando os efeitos deletérios da disfunção nos pacientes portadores.

Segundo Fronchetti et al. $^{13}$ a frequência cardíaca de repouso diminuiu com o treinamento físico.

Laterza et al. $^{14}$ em estudo de revisão, inferiram que o treinamento físico pode promover importantes adaptações neurovasculares em pacientes com hipertensão arterial sistêmica.

Estudos controlados têm demonstrado que a atividade física como reabilitação na disfunção autonômica de doentes com neuropatia autonômica diabética promove benefícios sistêmicos, aguda e cronicamente, o que faz do treinamento físico uma conduta eficaz para o tratamento destes pacientes. $^{15}$

Levando-se em consideração os prejuízos na qualidade de vida que o portador de disfunção autonômica apresenta, e sabendo das limitações e dificuldades do tratamento clínico, torna-se necessário verificar se práticas terapêuticas, tais como a reabilitação cardíaca podem trazer resultados mais favoráveis e duradouros ao paciente.

Diante disso o objetivo deste estudo foi verificar a eficácia do tratamento de reabilitação cardíaca na disfunção autonômica.

\section{METODOLOGIA}

Foi realizada uma revisão de literatura sistemática, comparativa e descritiva. Foram analisados artigos científicos sobre os efeitos da reabilitação cardíaca na disfunção autonômica. Para inclusão na amostra foram utilizados periódicos de livre acesso, disponíveis nas bases de dados científicas Medline, Lilacs, Pubmed e Scielo, disponíveis nas línguas portuguesa e inglesa.

Para busca foram utilizados os descritores reabilitação cardíaca; disautonomia; sistema nervoso autônomo e seus respectivos em inglês cardiac rehabilitation; dysautonomia; autonomic nervous system.

\section{RESUTADOS E DISCUSSAO}

Vários pesquisadores demonstraram os efeitos da disautonomia em diversas patologias, enquanto outros evidenciaram a influência de diferentes posições corpóreas bem como faixa etária, e a importância da ação da atividade física sobre os ajustes autonômicos. ${ }^{12-23}$ Os estudos relacionados à análise da Variabilidade da $\mathrm{FC}$ em diferentes métodos de avaliação ou tratamento foram estratificados quanto a população, diagnóstico, protocolo de avaliação ou tratamento e resultados, conforme as Tabelas 1, 2 e 3, divididas segundo o período de publicação dos estudos.

Tabela 1. Estudos relacionados à análise da variabilidade da frequência cardíaca em diferentes métodos de avaliação ou tratamento (2002 a 2006)

\begin{tabular}{|c|c|c|c|c|c|}
\hline Autor & Ano & População & Diagnóstico & $\begin{array}{l}\text { Protocolo de } \\
\text { Avaliação ou } \\
\text { Tratamento }\end{array}$ & Resultados \\
\hline Silva ${ }^{17}$ & 2002 & $\begin{array}{l}10 \text { homens, } \\
\text { ativos, saudáveis, } \\
\text { jovens }(23,7 \pm \\
3,02 \text { anos })\end{array}$ & Ausente & $\begin{array}{l}\text { Eletromiografia durante o } \\
\text { teste de físico tipo rampa e } \\
\text { correlação com repouso, } \\
\text { posição supino, sentado e } \\
\text { no exercício (incrementos } \\
20 \text { Watt/min). }\end{array}$ & $\begin{array}{l}\text { Alteração vago-simpático } \\
\text { com predomínio vagal em } \\
\text { supino. Associação entre } \\
\text { incremento de potências } \\
\text { durante exercício físico, } \\
\text { aumento FC e diminuição } \\
\text { VFC. }\end{array}$ \\
\hline $\begin{array}{l}\text { Brunetto et } \\
\text { al. }^{23}\end{array}$ & 2005 & $\begin{array}{c}41 \text { adolescentes } \\
\text { saudáveis ( } 22 \\
\text { homens e } 19 \\
\text { mulheres) com } \\
\text { idades entre } 15 \text { e } \\
18 \text { anos }\end{array}$ & Ausente & $\begin{array}{lr}\text { Teste de esforço máximo } \\
\text { em esteira com } \\
\text { eletrocardiograma, com } \\
\text { ergoespirometria } \\
\text { associada. }\end{array}$ & $\begin{array}{l}\text { Não foi detectado } \\
\text { nenhuma correlação no } \\
\text { grupo em estudo. }\end{array}$ \\
\hline $\begin{array}{l}\text { Fronchetti et } \\
\text { al. }{ }^{13}\end{array}$ & 2006 & $\begin{array}{c}20 \text { indivíduos } \\
\text { jovens } \\
(21,3 \pm 2,6 \text { anos })\end{array}$ & Ausente & $\begin{array}{l}\text { Verificar associação da FC } \\
\text { repouso, variabilidade FC } \\
\text { repouso e intensidade } \\
\text { esforço referente ao limiar } \\
\text { da VFC. }\end{array}$ & $\begin{array}{l}\text { FC repouso diminuiu com } \\
\text { treino. }\end{array}$ \\
\hline
\end{tabular}

Legenda: FC (Frequência Cardíaca); VFC (Variabilidade da Frequência Cardíaca) 
Recebido: 07 junho 2018. Aceito: 14 dezembro 2018. Revista Amazônia Science \& Health - 2018 Out/Dez.
Costa, GS. Cruz, HA. Rezende, AAB. Moreira, RF. Rodrigues, ESR. REABILITAÇÃO CARDÍACA NA DISFUNÇÃO AUTONÔMICA: ESTUDO DE REVISÃO

Tabela 2. Estudos relacionados à análise da variabilidade da frequência cardíaca em diferentes métodos de avaliação ou tratamento (2007)

\begin{tabular}{|c|c|c|c|c|c|}
\hline Autor & Ano & População & Diagnóstico & $\begin{array}{l}\text { Protocolo de } \\
\text { Avaliação ou } \\
\text { Tratamento }\end{array}$ & Resultados \\
\hline $\begin{array}{l}\text { Harthmann } \\
\text { et al. }\end{array}$ & 2007 & $\begin{array}{l}\text { Adultos de } 30 \text { a } \\
69 \text { anos }\end{array}$ & $\begin{array}{c}\text { Diabetes } \\
\text { Mellitus/ } \\
\text { Neuropatia } \\
\text { autonômica }\end{array}$ & $\begin{array}{l}\text { Manobra de Valsalva/ } \\
\text { Ortostatismo / Respiração } \\
\text { profunda Treinamento físico } \\
\text { com exercícios com } \\
\text { duração de cinco meses. }\end{array}$ & $\begin{array}{l}\text { Benefícios cardiovasculares, } \\
\text { metabólicos e autonômicos } \\
\text { após o exercício físico agudo } \\
\text { e crônico (eficaz conduta } \\
\text { não-farmacológica } \\
\text { diabetes). }\end{array}$ \\
\hline $\begin{array}{l}\text { Lopes } \\
\text { al. }{ }^{2}\end{array}$ & 2007 & $\begin{array}{c}32 \text { homens } \\
\text { sadios, } \\
\text { sedentários e } \\
\text { não-tabagistas, } \\
\text { sendo } 10 \text { jovens } \\
\text { e } 22 \mathrm{Ml} \\
\end{array}$ & Ausente & 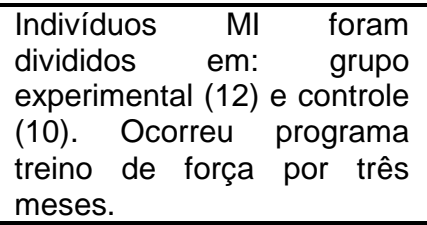 & $\begin{array}{l}\text { Aumento da idade altera } \\
\text { modulação autonômica sobre } \\
\text { nodo sinusal (diminuição } \\
\text { VFC não modificada pelo tipo } \\
\text { de treinamento). }\end{array}$ \\
\hline $\begin{array}{l}\text { Pantoni et } \\
\text { al. }^{19}\end{array}$ & 2007 & $\begin{array}{l}9 \text { indivíduos com } \\
\text { DPOC ( } 70 \text { anos) } \\
\text { e } 8 \text { saudáveis } \\
\text { (68 anos) }\end{array}$ & $\begin{array}{l}\text { DPOC e } \\
\text { saudáveis }\end{array}$ & $\begin{array}{l}\text { Eletrocardiograma para } \\
\text { registros durante 360s nas } \\
\text { posturas supina e sentada. }\end{array}$ & $\begin{array}{l}\text { DPOC: redução VFC c/ } \\
\text { diminuição simpática e vagal. } \\
\text { Sem ajustes autonômicos à } \\
\text { mudança postural, assim } \\
\text { como os idosos saudáveis. }\end{array}$ \\
\hline
\end{tabular}

Legenda: MI (Meia-idade); VFC (Variabilidade da Frequência Cardíaca); DPOC (Doença pulmonar obstrutiva crônica)

Tabela 3. Estudos relacionados à análise da variabilidade da frequência cardíaca em diferentes métodos de avaliação ou tratamento (2008 a 2011)

\begin{tabular}{|c|c|c|c|c|c|}
\hline Autor & Ano & População & Diagnóstico & $\begin{array}{l}\text { Protocolo de } \\
\text { Avaliação ou } \\
\text { Tratamento }\end{array}$ & Resultados \\
\hline $\begin{array}{l}\text { Zuttin } \\
\text { et al. }{ }^{16}\end{array}$ & 2008 & $\begin{array}{c}20 \text { homens } \\
\text { saudáveis e } \\
\text { sedentários }(22,6 \pm \\
2,5 \text { anos })\end{array}$ & Ausente & $\begin{array}{l}\text { Eletrocardiograma em repouso e nas } \\
\text { mudanças posturais. }\end{array}$ & $\begin{array}{lr}\text { Mudança } & \text { postural } \\
\text { provoca } & \text { ajustes } \\
\text { autonômicos } & \text { sobre } \\
\text { controle da FC. } & \end{array}$ \\
\hline $\begin{array}{l}\text { Reis } \\
\text { et al. }\end{array}$ & 2010 & $\begin{array}{c}28 \text { homens: } 10 \mathrm{c} / \\
\text { DPOC (GD) e } 69 \pm 9 \\
\text { anos; } 9 \mathrm{c} / \mathrm{ICC}(\mathrm{Gl}) \\
\text { e } 62 \pm 8 \text { anos; e } 9 \\
\text { saudáveis (GC) c/ } \\
64 \pm 5 \text { anos }\end{array}$ & $\begin{array}{l}\text { DPOC ou ICC } \\
\text { ou Saudáveis }\end{array}$ & $\begin{array}{l}\text { Eletrocardiograma em repouso e } \\
\text { supino: 1) } 15 \text { min na posição supino } \\
\text { e 2) } 4 \text { min durante M-ASR na } \\
\text { posição supino. }\end{array}$ & $\begin{array}{lr}\text { DPOC } & \text { e } \\
\text { produzem } & \text { ICC } \\
\text { negativo } & \text { sobre } \\
\text { controle } & \text { autonômico } \\
\text { da FC. } & \end{array}$ \\
\hline $\begin{array}{l}\text { Lima } \\
\text { et al. }\end{array}$ & 2011 & 15 homens jovens & Ausente & $\begin{array}{l}\text { Três sessões aleatórias: controle, } \\
\text { exercício-força } 50 \% \text { 1-RM e e } \\
\text { exercício-força } 70 \% \text { 1-RM. Cinco } \\
\text { exercícios/sessão p/ tronco e MMSS, } \\
\text { em } 3 \text { séries (12, } 9 \text { e } 6 \text { repetições). } \\
\text { FC aferida antes e aos } 20 \text { e } 50 \text { min } \\
\text { após as intervenções. }\end{array}$ & $\begin{array}{lr}\text { Maior } & \text { intensidade } \\
\text { exercício de } & \text { força } \mathrm{p} / \\
\text { tronco e } & \text { MMSS } \\
\text { promoveu } & \text { maior } \\
\text { aumento simpatovagal } \\
\text { cardíaco } & \text { pós- } \\
\text { exercício. } & \end{array}$ \\
\hline
\end{tabular}

Legenda: FC (Frequência Cardíaca); RM (Repetição máxima); MMSS (Membros superiores); M-ASR (Manobra de acentuação da arritmia sinusal respiratória); DPOC (Doença pulmonar obstrutiva crônica); ICC (Insuficiência cardíaca congestiva)

A regulação autonômica é fundamental para a ocorrência saudável de atividades cotidianas, laborais e desportivas.

Na pesquisa de Zuttin et al. ${ }^{16}$ ao avaliarem 20 homens jovens, saudáveis e sedentários, submetidos à avaliação por eletrocardiograma, para verificar o efeito da postura corporal sobre a modulação autonômica da frequência cardíaca em repouso, encontraram que, com a mudança postural, ocorreram ajustes autonômicos do sistema nervoso parassimpático e simpático sobre o controle da frequência cardíaca. Isso enfatiza a importância da integridade do sistema neurocárdico para a execução das atividades funcionais diárias em resposta às mudanças de posição corpórea. 
Recebido: 07 junho 2018. Aceito: 14 dezembro 2018. Revista Amazônia Science \& Health - 2018 Out/Dez.
Costa, GS. Cruz, HA. Rezende, AAB. Moreira, RF. Rodrigues, ESR. REABILITACÃO CARDÍACA NA DISFUNÇÃO AUTONÔMICA: ESTUDO DE REVISÃO
O estudo dirigido por Silva $^{17}$ utilizou a eletromiografia de superfície para verificar a variabilidade da frequência cardíaca (VFC) em resposta muscular frente ao exercício físico. Com o objetivo de avaliar o controle autonômico cardíaco a partir das respostas da frequência cardíaca e de sua variabilidade nas condições de repouso, nas posições supina e sentada, e durante teste de esforço físico dinâmico contínuo do tipo rampa (TEFDC-R), e para avaliar a atividade eletromiográfica no TEFDC-R e verificar a correlação entre a atividade eletromiográfica e a variabilidade da frequência cardíaca durante o TEFDC-R, foram estudados 10 homens, ativos, saudáveis, jovens em repouso, nas posições supina e sentada, e durante TEFDC-R, com incrementos de $20 \mathrm{~W} / \mathrm{min}$. Concluíram que houve uma alteração no balanço vago-simpático com predominância vagal na posição supina quando comparada com a sentada. Mostram ainda, uma associação entre 0 incremento de potências durante o exercício físico com o aumento do recrutamento de fibras musculares com concomitante aumento da frequência cardíaca e diminuição da VFC. Essa associação é atribuída tanto ao comando de controle central como dos mecanismos reflexos periféricos, ativados a partir das aferências das fibras musculares do grupo III (ergoceptores) e do grupo IV (metaboloreceptores). O que sugere o trânsito de informações advindos do córtex motor ativando as fibras musculares e paralelamente a área cardiovascular, modulando o sistema nervoso autonômico do coração, o que contribui para a homeostase simpatovagal em resposta às demandas físicas exigidas.

Atualmente sabe-se que a disautonomia pode comprometer a qualidade de vida dos portadores devido a seus sinais e sintomas associados. Várias patologias e outras condições, tais como faixa etária podem predispor à alteração da regulação autonômica.

Segundo o estudo de Grubb e Jorge ${ }^{18}$ é importante 0 destaque para um dos sintomas mais impactantes das atividades cotidianas dos portadores de disautonomia, a síncope vasovagal, que causa hipotensão e intolerância ortostática, o que pode gerar transtornos à qualidade de vida.

$\mathrm{Na}$ pesquisa de Pantoni et al. ${ }^{19}$ buscaram avaliar a variabilidade da frequência cardíaca (VFC) de idosos com doença pulmonar obstrutiva crônica (DPOC) e de idosos saudáveis frente à mudança postural. Para tanto, foram estudados 9 indivíduos com DPOC (70 anos) e 8 saudáveis (68 anos). Foi utilizado eletrocardiograma para registros durante 360 s nas posturas supina e sentada. Detectou-se que portadores de DPOC apresentaram redução da VFC com diminuição da atividade simpática e vagal e não apresentaram ajustes autonômicos frente à mudança postural, assim como os idosos saudáveis. O que reforça o prejuízo de capacidade de ajuste simpatovagal em detrimento da idade avançada e da presença de comorbidades.

Reis et al. ${ }^{20}$ encontraram resultados semelhantes aos de Patoni et al. ${ }^{19}$. Em seu estudo ao avaliar a modulação autonômica da frequência cardíaca (FC) em repouso, na postura supina e durante a manobra de acentuação da arritmia sinusal respiratória (M-ASR) de pacientes com doença pulmonar obstrutiva crônica (DPOC) ou com insuficiência cardíaca crônica (ICC). Para avaliação foram investigados 28 homens, sendo 10 (DPOC e $69 \pm 9$ anos), 9 (ICC e 62 \pm 8 anos), e 9 (saudáveis com $64 \pm 5$ anos). Foi obtido sinal eletrocardiográfico em repouso, 15 minutos na posição supina e quatro minutos durante M-ASR na posição supina. Concluíram que tanto a DPOC como a ICC produzem impacto negativo sobre 0 controle autonômico da FC.

Além da presença de comorbidades, mudanças corporais e idade avançada, o tipo de treino também pode influenciar na percepção da resposta que mantêm o equilíbrio simpatovagal.

Sabe-se que o treinamento físico pode contribuir para uma melhor regulação do sistema simpático e parassimpático, porém não foram observadas evidências favoráveis em todas as modalidades de treino ou mesmo em alguns métodos avaliativos.

No estudo de Lopes et al. ${ }^{12}$ investigaram a modulação autonômica exercida sobre o nodo sinusal, por meio da análise da variabilidade da frequência cardíaca (VFC), em indivíduos jovens e de meia-idade, bem como os efeitos de um programa de treinamento de força e resistência em indivíduos de meia-idade. Foram avaliados 32 homens sadios, sedentários e não-tabagistas, sendo 10 jovens e 22 de meia-idade, onde os mesmos foram submetidos à aquisição do sinal de eletrocardiograma para análise da VFC. O grupo meia-idade apresentou redução significativa, em comparação ao jovem, de todas as variáveis utilizadas para a investigação da VFC e o treinamento promoveu aumento significativo da força e resistência muscular em todos os grupamentos musculares. Ao término do estudo, verificou-se que os achados deste estudo confirmam que o aumento da idade provoca alteração na modulação autonômica exercida sobre o nodo sinusal e não houveram alterações significativas na modulação autonômica cardíaca em resposta ao programa de exercícios físicos realizados.

Possivelmente o tipo de treino pode ter influenciado nos resultados, uma vez que o treino de endurance é reconhecido por promover melhorias e ajustes cardiovasculares mais impactantes sobre a saúde e qualidade de vida. ${ }^{21}$ 
Recebido: 07 junho 2018. Aceito: 14 dezembro 2018. Revista Amazônia Science \& Health - 2018 Out/Dez.
Costa, GS. Cruz, HA. Rezende, AAB. Moreira, RF. Rodrigues, ESR. REABILITACÃO CARDÍACA NA DISFUNÇÃO AUTONÔMICA: ESTUDO DE REVISÃO
São bem difundidos os efeitos agudos e principalmente crônicos da reabilitação cardíaca. Os exercícios promovem benefícios fisiológicos, metabólicos, psicológicos, principalmente associados ao treinamento de endurance. Ocorre aprimoramento na função cardiovascular e respiratória.

Ocorre aumento da captação máxima de oxigênio; exige-se menor ventilação para qualquer intensidade submáxima; exige-se menor custo de oxigênio miocárdico para intensidades submáximas de esforço; ocorre redução de frequência cardíaca e pressão arterial (sistólica e diastólica) de repouso; aumenta o débito cardíaco pelo aumento do volume de ejeção sistólico e redução da frequência cardíaca; observa-se hipertrofia excêntrica (o que melhora a complacência ventricular e aumenta o volume plasmático); ocorre redução das catecolaminas; melhora a sensibilidade muscular à insulina. Além disso ocorre redução dos níveis do hormônio antidiurético (ADH); ocorre melhora da tolerância aos estímulos associados ao esforço (níveis crescentes de $\beta$-endorfina, $\beta$-lipotrofina e dinorfina geram sensação de prazer e contentamento pós-exercício); aumenta a sensação psicológica de bem-estar, o que gera alterações positivas no humor, redução das taxas de depressão clínica, e age sobre níveis ansiolíticos; melhora a densidade capilar no músculo esquelético; aumenta o limiar do exercício para acúmulo de lactato sanguíneo, aumenta o limiar do exercício para início de sinais e sintomas de doenças.

Ocorre ainda melhora nos níveis de colesterol lipoprotéico de alta densidade (HDL) e redução nos níveis séricos de triglicerídeos (reduz gordura corporal total, reduz gordura intra-abdominal, reduz a necessidade de insulina e aprimora a tolerância à glicose). Tais efeitos reduzem a morbimortalidade cardiovascular e melhora o desempenho nas atividades laborativas, recreativas e desportivas. ${ }^{21,22}$

Apesar da intensa repercussão hemodinâmica e cardiovascular em resposta aos exercícios físicos, nem sempre consegue-se comprovar os ajustes simpatovagais.

O método de avaliação empregado também pode influenciar na capacidade de confirmar as alterações hemodinâmicas fisiológicas e até mesmo patológicas do sistema autonômico durante exercícios ou mesmo em repouso.

Em outro estudo Brunetto et al. ${ }^{23}$ compararam - limiar de variabilidade da frequência cardíaca e o primeiro limiar ventilatório, em uma amostra de adolescentes (41 sujeitos, sendo 22 rapazes e 19 moças, com idades entre 15 e 18 anos) submetidos a teste de esforço físico de carga máxima em esteira ergométrica, com ergoespirometria associada. A variabilidade da frequência cardíaca foi analisada por intermédio dos intervalos $R-R$, através de plotagem. Neste estudo não foi possível correlacionar o limiar de variabilidade da frequência cardíaca e o primeiro limiar ventilatório em adolescentes, embora acredita-se que haja uma associação entre ambos fatores.

A reabilitação física em portadores de disautonomia pode contribuir favoravelmente para minimizar os sintomas deletérios da doença.

$\mathrm{O}$ estudo de Fronchetti et al. ${ }^{13}$ encontraram uma melhoria significativa na modulação autonômica em resposta ao treinamento físico. Ao verificar o grau de associação entre a frequência cardíaca de repouso, diferentes índices de variabilidade da frequência cardíaca em repouso e a intensidade de esforço referente ao limiar de variabilidade da FC em indivíduos jovens, os pesquisadores observaram que a frequência cardíaca de repouso diminuiu com o treinamento.

Tais achados indicam que elevada atividade vagal de repouso pode contribuir para retardar o aumento da predominância simpática em intensidades progressivas de esforço. $O$ que confirma o papel positivo do treinamento físico sobre o controle autonômico.

Posteriormente, em um estudo realizado em 2011, Lima et $\mathrm{al}^{24}$ tiveram como objetivo analisar o efeito agudo da intensidade do exercício de força para tronco e membros superiores na modulação autonômica pós-exercícios, em 15 homens jovens. Foram realizadas três sessões em ordem aleatória, sendo controle (C), exercício de força com $50 \%$ de 1- RM (E50\%) e exercício de força com $70 \%$ de 1 RM (E70\%). As sessões incluíram cinco exercícios para tronco e membro superior, realizados em três séries de 12, 9 e 6 repetições, respectivamente. A frequência cardíaca foi obtida para avaliação de sua variabilidade, antes dos exercícios aos 20 minutos e 50 minutos após. Nesse estudo concluíram que a maior intensidade de exercício de força para tronco e membros superiores promoveu, agudamente, maior aumento do balanço simpatovagal cardíaco pós-exercício.

Os pesquisadores Harthamann et al. $^{15}$ ao levantarem os efeitos da atividade física na disfunção autonômica de pacientes com neuropatia autonômica, observaram que os benefícios cardiovasculares, metabólicos e autonômicos após - exercício físico agudo e crônico, tornam a reabilitação física uma eficaz conduta nãofarmacológica para o tratamento de pacientes com diabetes.

Da mesma forma Laterza et al. ${ }^{14}$ em estudo de revisão afirmaram que o treinamento físico pode promover importantes adaptações neurovasculares em hipertensos. Sendo assim, a prática regular de exercícios físicos atua como uma das condutas nãomedicamentosas mais eficazes para o controle da hipertensão arterial. 
Recebido: 07 junho 2018. Aceito: 14 dezembro 2018. Revista Amazônia Science \& Health - 2018 Out/Dez.
Costa, GS. Cruz, HA. Rezende, AAB. Moreira, RF. Rodrigues, ESR. REABILITACCÃO CARDÍACA NA DISFUNÇÃO AUTONÔMICA: ESTUDO DE REVISÃO

\section{CONCLUSAO}

Infere-se que a reabilitação cardíaca em pacientes com disfunção autonômica pode minimizar os sintomas associados à doença e pode contribuir para a melhoria na qualidade de vida destes pacientes.

Entretanto sugere-se novos estudos para ampliar os conhecimentos sobre o papel da reabilitação física e sua influência na modulação autonômica.

\section{REFERÉNCIAS}

1. Natelson BH. Neurocardiology: an interdisciplinary area for the 80s. A.M.A Arch Neurol.1985; 42(2):178-184.

2. Junqueira Jr LF. Disfunção autonômica cardíaca. Doenças do coração- tratamento e reabilitação. Ed. Porto CC: Rio de Janeiro. 1998; (58): 306-11.

3. Angelis K, Santos MSB, Irigoyen MC. Sistema nervoso autônomo e doença cardiovascular. RBM Cardiol. 2004; (3): 1-7.

4. Lombardi F, Sandrone G, Pernpruner S, Sala R, Garimoldi M, Cerutti S, Malliani A. Heart rate variability as an index of sympathovagal interaction after acute myocardial infarction. Am J Cardiol. 1987; 60(16): 1239-1245.

5. Rocha EA. Síndromes neuralmente mediadas. Arq Bras Cardiol. 2006; 87(3): 34-44.

6. Elias, NJ. Avaliação diagnóstica da insuficiência autonômica. RELAMPA Rev Latinoam Marcapasso Arritmia. 2016; 29(2): 63-72.

7. Williams CE, Steven KN. Vocal correlates of emotional states in Darby. Ed. JK Speech evaluation in psychiatry - Grune \& Stratton: Inc; 1981.

8. Bassich CJ, Ludlow CL, Polinsky RJ. Speech symptoms associated with early signs of Shy Drager syndrome. J Neurol Neurosurg Psychiartry. 1984; 47(9):995-1001.

9. Truong DD, Bhidayasiri R, Wolters E. Management of non-motor symptoms in advanced Parkinson disease. J Neurol Sci. 2008; 266(1) : 216228.

10. Izdebski K. Erotic and Orgasmic vocalization: myth, reality or both. In: Emotion in the human voice. New York, 2008.

11. Mostarda C, Wichi R, Sanches IC, Rodrigues B, Angelis K, Irigoyen MC. Hipertensão e modulação autonômica no idoso: papel do exercício físico. Rev Bras Hipertens. 2009; 16(1): 55-60.

12. Lopes, FL, Pereira FM, Reboredo MM, Castro TM, Vianna, JM, Novo JRJM, Silva LP. Redução da variabilidade da frequência cardíaca em indivíduos de meia-idade e o efeito do treinamento de força. Rev Bras Fisiot. 2007; 11(2): 113-119.

13. Fronchetti L, Nakamura F, Aguiar C, Oliveira F. Indicadores de regulação autonômica cardíaca em repouso e durante exercício progressivo: aplicação do limiar de variabilidade da frequência cardíaca. Rev Port Ciênc Desporto. 2006; 6(1): 21-28.

14. Laterza MC, Amaro G, Negrão CE, Rondon MUPB. Exercício físico regular e controle autonômico na hipertensão arterial. Rev SOCERJ. 2008; 21(5): 320-328.

15. Harthmann ÂDA, Manfroi WC. Efeitos da atividade física na modulação autonômica em pacientes diabéticos com disfunção autonômica. Rev HCPA. Porto Alegre. 2007; 27 (3): 37-42.

16. Zuttin RS, Moreno MA, César MC, Martins LEB, Catai AM, Silva E. Avaliação da modulação autonômica da frequência cardíaca nas posturas supina e sentada de homens jovens sedentários. Rev Bras Fisiot. 2008; 12(1): 7-12.

17. Silva CS. Análise da modulação autonômica do coração em repouso e atividade eletromiográfica relacionada à variabilidade da frequência cardíaca durante o exercício dinâmico em cicloergômetro. São Paulo-SP. Tese de Doutorado. Universidade de São Paulo; 2002.

18. Grubb BP, Jorge CS. Aspectos da classificação, diagnóstico e tratamento das síndromes de disfunção autonômica associadas à intolerância ortostática. Arq Bras Cardiol. 2000; 74(6): 537-544.

19. Pantoni CBF, Reis MS, Martins LEB, Catai AM, Costa D, Borghi-Silva A. Estudo da modulação autonômica da frequência cardíaca em repouso de pacientes idosos com doença pulmonar obstrutiva crônica. Rev Bras Fisiot. 2007; 11(1): 35-41.

20. Reis MS, Deus AP, Simões RP, Aniceto IA, Catai AM, Borghi-Silva A. Controle autonômico da frequência cardíaca de pacientes com doenças cardiorrespiratórias crônicas e indivíduos saudáveis em repouso e durante a manobra de acentuação da arritmia sinusal respiratória. Rev Bras Fisiot. 2010; 14(2): 106-13.

21. American College of Sports Medicine. Diretrizes do ACSM para os testes de esforço e sua prescrição. 9a Ed. Rio de Janeiro, 2014. 
22. Neder JA, Nery LE; Fisiologia Clínica Do Exercício: Teoria e Prática. 1aㅡ. Ed. Artes Médicas: São Paulo, 2003.

23. Brunetto AF, Silva BM, Roseguini BT, Hirai DM, Guedes DP. Limiar ventilatório e variabilidade da frequência cardíaca em adolescentes. Rev Bras Ciênc Esport. 2005; 11(1): 22-7.

24. Lima AHRDA, Forjaz CLDM, Silva GQDM, Menêse AL, Silva AJMR, Ritti-Dias RM. Efeito agudo da intensidade do exercício de força na modulação autonômica cardíaca pós-exercício. Arq Bras Cardiol. 2011; 96(6): 498-503. 